\title{
Performance of Ant System over other Convolution Masks in Extracting Edge
}

\author{
A. Amali Asha \\ St.Xavier's College (Autonomous) \\ Palayamkottai - 627002, India
}

\author{
S.P. Victor \\ Department of Computer Science \\ St.Xavier's College (Autonomous) \\ Palayamkottai - 627 002, India.
}

\author{
A. Lourdusamy \\ Department of Mathematics \\ St.Xavier's College (Autonomous) \\ Palayamkottai - 627 002, India.
}

\begin{abstract}
The front end of most vision systems consists of edge detection as preprocessing. The vision of objects is easy for the human because of the natural intelligence of segmenting, pattern matching and recognizing very complex objects. But for the machine, everything needs to be artificially induced and it is not so easy to recognize and identify objects. Towards Computer vision, the Machine needs pattern recognition; extracting the important features so as to recognize the objects, where the boundary detection or the edge detection is very crucial. Edge detection is finding the points where there are sudden changes in the intensity values and linking them suitably. This paper aims, at presenting a comparative study on the Gradient based edge detectors with a swarm intelligence. Though, these detectors are applied on to the same image, they may not see the same edge pixels. Some detectors seems to be good only for simple transparent images which are less noise prone, and marks pseudo and congested edges in case of denser images. Hence it would be appreciated, to have an edge detector, which is sensitive in detecting edges in majority of the common types of edges. With this in mind, the authors propose a new edge detector based on swarm intelligence, which fairly detects the edges of all types of images with improved quality, and with a low failing probability in detecting edges.
\end{abstract}

\section{Key words:}

Edge, Segmentation, Feature Extraction, Swarm intelligence, Ant System

\section{INTRODUCTION}

Computer vision aims to duplicate the effect of human vision by electronically perceiving and understanding an image. Giving computers the ability to see is not an easy task. Towards computer vision the role of edge detection is very crucial as it is the preliminary or fundamental stage in pattern recognition. Edges characterize object boundaries and are therefore useful for segmentation and identification of objects in a scene. The idea that the edge detection is the first step in vision processing has fueled a long term search for a good edge detection algorithm.

Swarm intelligence methods are computational methods inspired by animals such as social insects acting together to solve complex problems. The main application of these techniques has been to combinatorial optimization problems. This paper discusses work-in-progress on the application of swarm intelligence ideas to image processing problem, such as extracting boundaries or edges of objects. This paper presents an Ant Colony Optimization based mechanism to extract the edges in an image. Experimental results indicate that the proposed method is more efficient than the Gradient based edge detection techniques

\section{EXTRACTING EDGES FROM IMAGES}

An edge [1], [2], [3] is a jump in Intensity or otherwise it can be considered as a typical boundary between two dissimilar regions. An edge is not a physical entity, just like a shadow. It is where the picture ends and the wall starts. It is where the vertical and the horizontal surfaces of an object meet. It's what happens between a bright window and a dark. Edges in images are areas with strong intensity contrasts.

\subsection{The Edge Structure}

If we look at the concept of a digital edge a little closer, an edge is a set of connected pixels that lie on the boundary between two regions. An ideal edge is a set of connected pixels, in the vertical direction, each of which is located at an orthogonal step transition in gray level. In practice the imperfections in image acquisition yield edges that are blurred, with the degree of blurring being determined by factors such as the quality of the image acquisition system, the sampling rate, and illumination conditions under which the image is acquired. Effects such as refraction or poor focus can result in objects with boundaries defined by a gradual change in intensity.

As a result, if we closely observe the cross section of the edge it is nothing but the shape of the ramp. An ideal edge is a ramp with an infinite slope. The slope of the ramp is inversely proportional to the degree of blurring in the edge. In this model, we no longer have a thin ( one pixel thick) path. Instead, an edge point now is any point contained in the ramp, and an edge would then be "a set of such points that are connected. The "thickness" of the edge is determined by the length of the ramp, as it transitions from an initial to a final gray level. This length is determined by the slope, which, in turn, is determined by the degree of blurring. Blurred edges tend to be thick and sharp edges tend to be thin.

\subsection{Edge Detection Categories}

Though, a variety of edge detection Techniques are available, the most of them may be grouped into two categories, Gradient and Laplacian [2]. The gradient method detects edges by looking for a maximum and minimum in the first derivatives of the images [2] ie, it assumes a local maximum at an edge. The laplacian method searches for zero crossing in the second derivatives of the image to find the edges [2]. In gradient method for a continuous image say $\mathrm{f}(\mathrm{x}, \mathrm{y})$ we consider the two edge directions; horizontal and vertical represented by $\partial \mathrm{x}(\mathrm{f}(\mathrm{x}$, $y)$ ) and $\partial \mathrm{y}(\mathrm{f}(\mathrm{x}, \mathrm{y}))$. The gradient vector points in the direction of maximum rate of change of ' $\mathrm{f}$ ' at co-ordinates $\mathrm{f}(\mathrm{x}, \mathrm{y})$. The 
important quantities in edge detection are the gradient magnitude denoted by [6]

$\nabla f(x, y)=\sqrt{\left(\hat{\partial x}(f(x, y))^{2}+\left(\hat{\partial y}(f(x, y))^{2}\right.\right.}$

and the gradient orientation ( or) the direction of the gradient vector denoted as

$\propto(x, y)=\tan -1 \sqrt{(\hat{\partial y}(f(x, y)) /(\hat{\partial x}(f(x, y))}$

where the angle is measured with respect to the $\mathrm{x}$-axis. The direction of an edge at $\mathrm{x}, \mathrm{y}$ is perpendicular to the direction of the gradient vector at that point. A pixel location is declared as an edge location if the gradient magnitude exceeds some threshold.

\subsection{Threshold and edge linking}

We are led to the idea that, to be classified as a meaningful edge point, the transition in gray level associated with that point has to be significantly stronger than the background at that point. Since we are dealing with local computations, the method of choice to determine whether a value is "significant" or not is to use a threshold. Thus, we define a point in an image as being an edge point if its two-dimensional first-order derivative is greater than a specified threshold. A set of such points are connected according to a predefined criterion of connectedness.

It is important to note that these definitions do not guarantee success in finding edges in an image. They simply give us a formalism to look for them. The choice of threshold value determines the resulting segmentation and hence the perceived quality of the edge detector. It is useful to consider the cumulative histogram of the gradient image in selecting the appropriate threshold value. The location of all edge points constructs an edge map. The selection of the threshold value is an important design decision that depends on a number of factors such as image brightness, contrast, noise level etc...A weak edge positioned between two strong edges is highly probable that this inter positioned weak edge should be a part of a resulting boundary. If, on the other hand, an edge (even a strong one) is positioned by itself with no supporting context, it is probably not a part of any border.

\subsection{Edge Detection Techniques}

Four frequently used methods are considered here for comparison. Edge detection operators [5], [6], [7] examine each pixel neighborhood and quantify the slope. There are several ways are available. Most of which are based upon convolution with a set of directional derivative masks.

\subsubsection{The Sobel Detection}

The Sobel operator [5], [6], [7] performs a 2D spatial gradient measurement on an image, hence emphasizes regions of high spatial frequency that correspond to edges. The sobel convolution mask is as shown in figure 1.

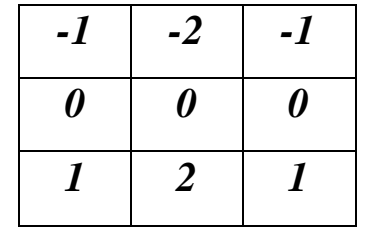

Fig 1 : Sobel mask

\subsubsection{The Prewitt Detection}

The Prewitt edge detection is an appropriate way to estimate the magnitude and orientation of an edge. The convolution mask of Prewitt [5], [6], [7] is as shown in Figure 2

\begin{tabular}{|l|l|l|}
\hline-1 & -1 & -1 \\
\hline 0 & 0 & 0 \\
\hline 1 & 1 & 1 \\
\hline
\end{tabular}

Fig 2 : Prewitt mask

\subsubsection{The Roberts Detection}

The Roberts edge detection is a local differential operator for finding edges. Roberts operator performs a 2D spatial measurement on an image. The mask value [5], [6], [7] is as shown in Figure 3.

\begin{tabular}{|l|l|}
\hline 1 & 0 \\
\hline 0 & -1 \\
\hline
\end{tabular}

Fig 3 : Roberts mask

\subsubsection{The Kirsch Detection}

In Kirsch edge detection each point in the image is convolved with eight masks. Each mask responds maximally to an edge oriented in a particular general direction. The mask value [5], [6], [7] is as shown in Figure 4.

\begin{tabular}{|l|l|l|}
\hline 5 & 5 & 5 \\
\hline-3 & 0 & -3 \\
\hline-3 & -3 & -3 \\
\hline
\end{tabular}

Fig 4 : Kirsch mask 


\section{THE ACO APPROACH}

Ant Colony Optimization (ACO) is a paradigm for designing metaheuristic algorithms for combinatorial optimization problems [8], [9]. In the early 1990's Ant Colony Optimization (ACO) was introduced by $\mathrm{M}$. Dorigo and Colleagues.

The inspiring source of ACO is the foraging behavior of real ants. Initially ants have no idea of where food is in the environment, when searching for food, ants initially explore the area surrounding their nest in a random manner. As soon as an ant finds a food source, it evaluates the quantity and the quality of the food and carries some of it wander back to the nest. During the return trip, the ant deposits a chemical substance called pheromone on the ground. The pheromone deposited varies in quantity depending upon the quantity and quality of the food. This will guide other ants to the food source.

The boundary is identified by considering the gray levels of nearest neighbors of the current position. The neighbors are identified from the current position by considering 8 connectivity as we did in the convolution mask methods. Each ant moves to an adjacent cell and reinforces the pheromone level on that spot. In order to move from state $i$ to $j$ the probability [11][12] is used as given in equation 3

$p i j(t)=\frac{[\tau i j]^{\alpha}[\eta i j]^{\beta}}{\sum j \varepsilon \Omega i[\tau i j]^{\alpha}[\eta i j]^{\beta}} \quad$ if $\mathrm{j} \varepsilon \Omega \mathrm{i}$

The value of $\tau_{i \tilde{y} j}$ is used for moving to adjacent cell which is given in equation 4

$$
\tau_{i j}=k+\frac{\sigma}{k+\delta \sigma}
$$

Where $\mathrm{k}$ is a constant

Similarly the factor ${ }^{\eta_{i \tilde{j}}}$ is given as in equation 5

$$
\eta_{i j}=\frac{V_{m}\left(I_{i j j}\right)}{V_{\max }}
$$

Where

$$
I_{\tilde{i} \tilde{j}} \text { is the current intensity value of pixel at } \mathrm{i}, \mathrm{j}
$$

$V_{\max }$ is the maximum intensity variation between pixels in the whole image. It is calculated based on the 8 direction from the current pixel is as shown in equation 6 and in figure 5 .

$$
V_{m}\left(I_{i, j}\right)=\left|I_{i-1, j-1}-I_{i+1, j+1}\right|+\left|I_{i-1, j}-I_{i+1, j}\right|+\left|I_{i-1, j+1}-I_{i+1, j-1}\right|+\left|I_{i, j-1}-I_{i, j+1}\right|
$$

When the ant moves from one pixel to another if that pixel falls on the edge then it should update the pheromone value of that pixel as given in equation 7 .

$$
P_{\text {update }}=P_{\text {old }}+\rho \nabla / 255
$$

Where $\nabla$ is the difference between the median gray levels of previous cell and its neighbors and current cell and its neighbor.

\subsection{Features}

- The first and most obvious is low error rate. It is important that edges occurring in images should not be missed and there should be no response to pseudo edges.

- The second criterion is localization.

- The third criterion is to have only one response to a single edge.

The simple threshold technique is used here to partition the image histogram by a single global threshold T, segmentation is then accomplished by scanning the image, pixel by pixel and labeling each pixel as edge point or not, depending on whether the gray level of that pixel is greater or less than the value of $\mathrm{T}$.

\subsection{Algorithm}

\begin{tabular}{|l|l|l|}
\hline $\begin{array}{l}\text { i- } 1, j- \\
1\end{array}$ & $i, j-1$ & $i+1, j-1$ \\
\hline$i-1, j$ & ANT & $i+1, j$ \\
\hline $\begin{array}{l}i- \\
1, j+1\end{array}$ & $i, j+1$ & $i+1, j+1$ \\
\hline
\end{tabular}

Fig 5 : 8 directions 
ten images were evaluated, of which the results of three sets are presented here.

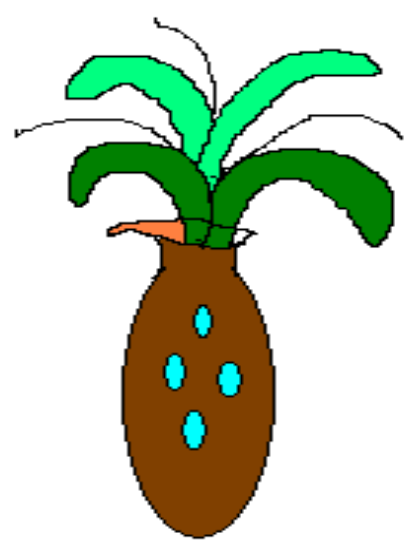

(a) Vase

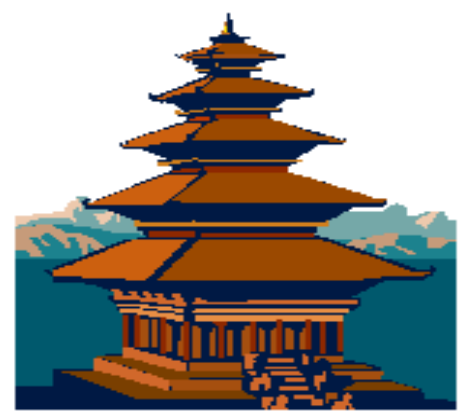

(b) Tower

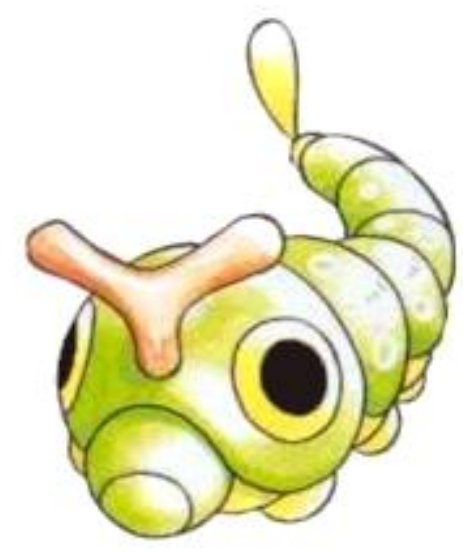

(c) Lart

Fig 6. Original Images
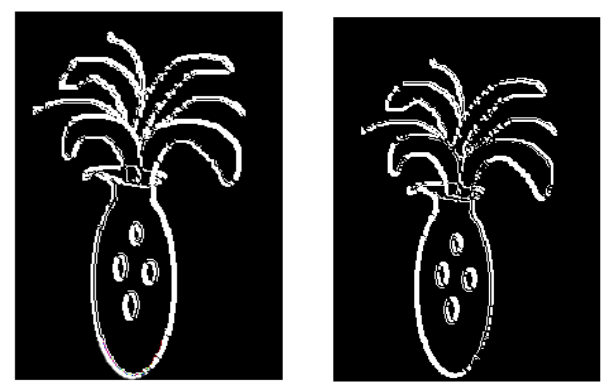

Sobel Detection

Prewitt Detection
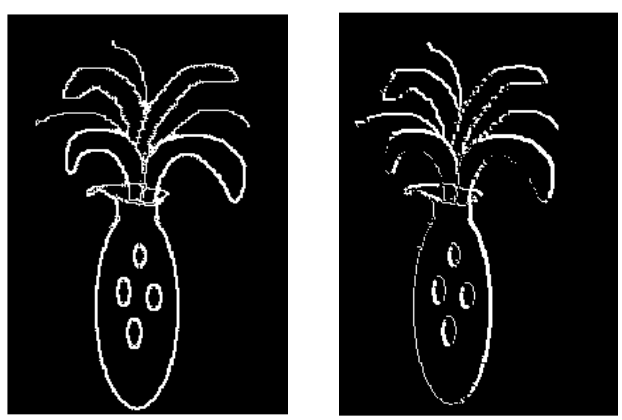

Kirsch Detection Roberts Detection

Fig 7. Edges in Flower Vase
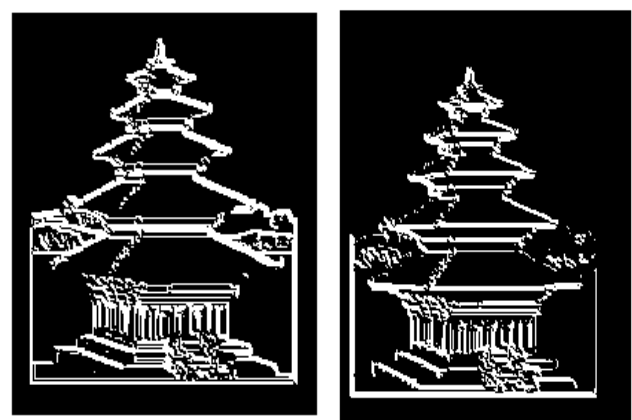

Sobel Detection Prewitt Detection
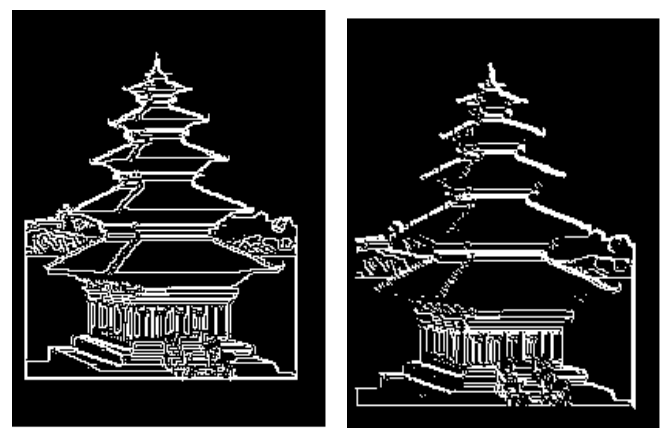

Kirsch Detection Roberts Detection

Fig 8. Edges in Tower 

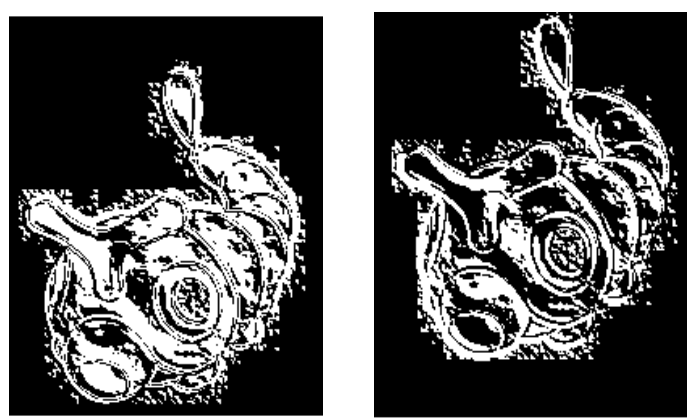

\section{Sobel DetectionPrewitt Detection}
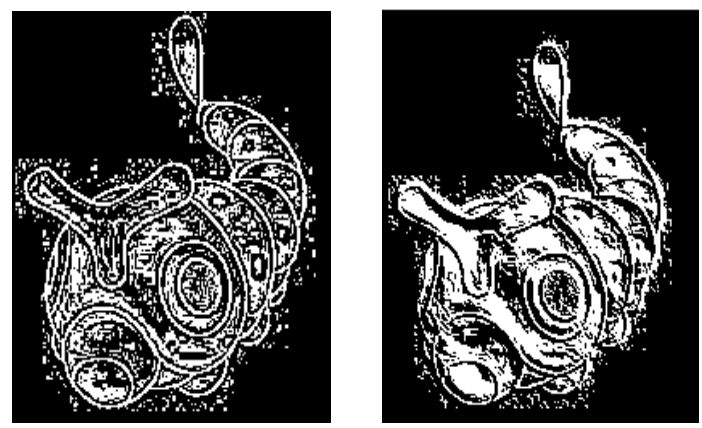

Kirsch Detection

Roberts Detection

Fig 9. Edges in Lart
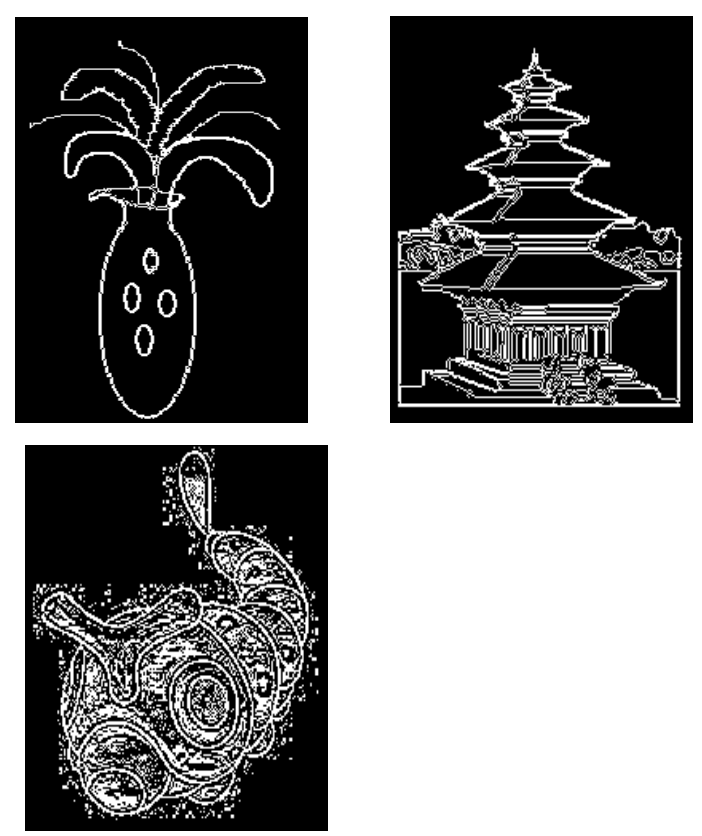

Fig 10. Edges using Ant System
Table 1. Edge Detection Comparison

\begin{tabular}{|l|l|l|l|}
\hline & $\begin{array}{l}\text { Flower } \\
\text { Vase }\end{array}$ & Tower & Lart \\
\hline SOBEL & $\begin{array}{l}\text { Prominent } \\
\text { discontinuitie } \\
\text { s \& very thick } \\
\text { Edges }\end{array}$ & $\begin{array}{l}\text { Discontinuit } \\
\text { ies \& thick } \\
\text { Edges }\end{array}$ & $\begin{array}{l}\text { Edges are not } \\
\text { clear. Noise } \\
\text { distortion } \\
\text { present }\end{array}$ \\
\hline PREWIT & $\begin{array}{l}\text { No } \\
\text { Discontinuity } \\
\text { but } \\
\text { Very thick } \\
\text { edges }\end{array}$ & $\begin{array}{l}\text { Discontinuit } \\
\text { ies \& Very } \\
\text { thick edges }\end{array}$ & $\begin{array}{l}\text { Edges are } \\
\text { identified but } \\
\text { Much noise } \\
\text { distortion }\end{array}$ \\
\hline ROBERTS & $\begin{array}{l}\text { Discontinuitie } \\
\text { s present but } \\
\text { Very thin and } \\
\text { clear edges }\end{array}$ & $\begin{array}{l}\text { Much } \\
\text { Discontinuit } \\
\text { ies } \\
\text { Thin and } \\
\text { clear edges }\end{array}$ & $\begin{array}{l}\text { Edges are } \\
\text { identified with } \\
\text { Little noise } \\
\text { distortion }\end{array}$ \\
\hline KIRSCH & $\begin{array}{l}\text { Less } \\
\text { Discontinuitie } \\
\text { s present but } \\
\text { Very thin and } \\
\text { clear edges }\end{array}$ & $\begin{array}{l}\text { Much less } \\
\text { discontinuiti } \\
\text { es } \\
\text { Thin and } \\
\text { clear edges }\end{array}$ & $\begin{array}{l}\text { Edges are clear } \\
\text { Noise distortion } \\
\text { present }\end{array}$ \\
\hline $\begin{array}{l}\text { Continuous \& } \\
\text { Very thin and } \\
\text { clear edges }\end{array}$ & $\begin{array}{l}\text { Much less } \\
\text { discontinuiti } \\
\text { es } \\
\text { Thin and } \\
\text { clear edges }\end{array}$ & $\begin{array}{l}\text { Edges are } \\
\text { clearly } \\
\text { identified } \\
\text { Little noise } \\
\text { distortion }\end{array}$ \\
\hline $\begin{array}{l}\text { ANT } \\
\text { SYSTEM }\end{array}$ & \multicolumn{2}{|l}{} \\
\hline
\end{tabular}

\section{CONCLUSION}

Subjective analysis reveals that the new approach using Ant System of edge detection is effective in all the three categories of the images selected. Edge detecting in an image significantly reduces the amount of data and filters out useless information while presenting the important structural properties in an image. Edge detection is difficult in noisy images since both the noise and the edges contain high frequency content. Better results can be obtained by applying a noise filter prior to the edge detection.

As the study is in its initial phase, the quality of the image is judged by subjective rating of human. Quantitative estimation of time and localization effects are under development. Also the study is carried out with limited images, and additional tests and statistical investigations are necessary. 


\section{REFERENCES}

[1]. Milan Sonka, Vaclav Hlavac, Roger Boyle Image Processing, Analysis and Machine Vision Thomson Asia Pte. Ltd, Singapore.

[2]. Rafael C. Gonzalez, Richard E.Woods, Digital Image Processing, Peterson Education Asia.

[3]. V.Hlavac, M. Sonka and R Boyle Image Processing Analysis and Machine Vision, Chappman and Hall 1993

[4]. D Marr E.Hildreth Thoery of Edge Detection Computer vision, Los Alamitos CA, 1991 pp 77- 107

[5]. N.Senthil Kumaran and R.Rajesh "Edge Detection Techniques for Image Segmentation - A survey" Proceedings of the International Conference on Managing Next Generation Software Applications (MNGSA - 08), 2008, pp 749-760
[6]. Mohamed Roushdy Comparative Study of Edge Detection Algorithms Applying on Grayscale Noisy Image Using Morphological Filter, GVIP Journal Volume 6, Dec-2006

[7]. Raman Maini and J.S.Sobel "Performance Evaluation of Prewitt Edge Detector for Noisy Images ", GVIP Journal Vol 6, Dec.2006.

[8]. DORIGO, M.-BLUMB, C. : Ant colony optimization theory: A survey, Theoretical computer Science, 2005 Elsevier BV.

[9]. Marco Dorigo, Vittorio Maniezzo and Alberto Colorni "The Ant System: Optimization by a colony of cooperating agents" IEEE Transactions on Systems, Man, and Cybernetics-Part B, Vol.26, No.1, 1996, pp.1-13 